\title{
Balance 1999
}

Carlos 0 ropeza-A búndez, Lic. en Cien. Pol., ${ }^{(1)}$ Mónica Fuentes-Ramírez, Lic. en Econ. ${ }^{(2)}$

$\mathrm{T}$ al como lo hemos venido haciendo desde hace un lustro, el primer número del nuevo volumen nos sirve como marco para realizar un balance de las actividades desarrolladas durante el año que termina. Además, durante 1999 Salud Pública de México cumplió 40 años de vida, por lo que en esta ocasión incluiremos algunos indicadores de los avances obtenidos en los años previos.

Para iniciar, mencionaremos algunos hechos relevantes sucedidos durante 1999. El primero se refiere a que, en atención a una de las principales recomendaciones emitidas por nuestro Comité Editorial en la reunión general que sostuvo en marzo de 1998, se revisó la estructura del Consejo de Editores y las secciones que lo componían. El resultado derivó en una reorganización que consistió en la aparición del área de Sociomedicina, junto con las que ya existían: Epidemiología y Métodos Cuantitativos, Sistemas de Salud y, finalmente, Biomedicina; además, se designaron responsables para las secciones de Indicadores, Noticias y Páginas de Salud Pública. Esta nueva estructura ha generado resultados positivos al proporcionar mayor consistencia al trabajo editorial.

Un segundo aspecto que podemos mencionar es que, además de continuar en diez sistemas de índices y bases de datos reconocidos internacionalmente, Salud Pública de México ha sido incluida en la biblioteca electrónica SciELO*-Salud Pública creada por el Centro Latinoamericano y del Caribe de Información en Ciencias de la Salud (Bireme), que permite tanto el acceso al texto completo como identificar las consultas y citaciones que se hacen de los artículos de la revista en las publicaciones que integran este sistema.

El otro punto relevante consiste en la creciente demanda que ha captado la revista por medio de su propia dirección electrónica. Lo anterior se pone de manifiesto al observar que los accesos a Salud Pública de México representaron más de $60 \%$ de los recibidos en la página electrónica del Instituto Nacional de Salud Pública (INSP). Debido a la importancia que esto tiene, en los próximos meses analizaremos estos datos para estar en mejores posibilidades de ofrecer información detallada a este respecto.

Los puntos anteriores muestran algunos de nuestros avances más importantes; pero aunado a ello, y como resultado del trabajo editorial continuo, también se obtuvo una alta productividad durante 1999, ya que por vez primera publicamos un volumen compuesto por ocho números, esto es, seis regulares y dos suplementos. De estos últimos, uno estuvo dedicado al problema de las enfermedades intrahospitalarias en nuestro país, y el otro, al tema Salud, genética y ambiente, para lo cual se reunieron trabajos de especialistas nacionales y extranjeros con el propósito de dar a conocer algunos avances y revisiones de investigación en esta área. En total, durante el año pasado publicamos 66 trabajos que fueron revisados por pares, de los cuales $82 \%$ corresponden a trabajos con resultados de investigaciones originales.

Lo anterior fue producto de un proceso activo en donde el número de trabajos en evaluación fue de 220 , de los cuales $53(24 \%)$ corresponden a trabajos reci-

* Scientific Electronic Library 0 nline

(1) Editor Ejecutivo de Salud Pública de México (SPM), Instituto N acional de Salud Pública (IN SP), México.

(2) Jefa del Departamento de Edición, SPM, IN SP, México. 
bidos en 1998, y 167 (76\%), a manuscritos nuevos; de estos últimos, 23 (14\%) fueron aceptados; 107 (64\%), rechazados, y $37(22 \%)$ terminarán su evaluación durante el 2000. Es importante resaltar que para la invaluable labor de revisión de estos trabajos, contamos con la colaboración de 161 especialistas, de los cuales la tercera parte (33\%) fueron mujeres y sólo 29\% del total correspondieron a investigadores del INSP. Es preciso señalar que a lo largo de este último lustro el promedio de dictaminadores se ha mantenido por arriba de los 164 investigadores por año.

Por otro lado, en el volumen que analizamos 277 investigadores participaron como autores, sólo en lo que respecta a los trabajos que previamente se sometieron a revisión por pares. El origen geográfico de los mismos fue el siguiente: $49 \%$ son de la ciudad de México; $32 \%$, de otros estados de la República mexicana, y $19 \%$, del extranjero; mientras que para el caso de primera autoría, exclusivamente, este indicador resultó en 46,36 y $18 \%$, respectivamente.

El detalle de la procedencia institucional de las autorías se puede observar en la figura 1, aunque cabe destacar que únicamente $23 \%$ de las primeras autorías correspondió a investigadores del INSP, proporción que se reduce a $14 \%$ si tomamos en cuenta el total de autorías. En este mismo rubro podemos señalar que sólo $8 \%$ de los trabajos publicados contó con autorías exclusivamente de investigadores del Instituto, mientras que en $73 \%$, estos últimos, no tuvieron ninguna participación (figura 2). Este aspecto resulta sumamente importante ya que, por lo regular, se considera que una revista de esta naturaleza generalmente responde a las necesidades de publicación de los investigadores pertenecientes a la institución responsable de su edición. En Salud Pública de México hemos reiterado constantemente nuestro compromiso con la pluralidad; en este sentido, a lo largo de los útimos cinco años, el porcentaje promedio de las primeras autorías de investigadores del INSP fue de $21 \%$, y se reduce a $20 \%$ para el caso del total de autores.

En lo que se refiere a género, $42 \%$ de los investigadores que publicaron en 1999 fueron mujeres, tanto para el total de autores como para el caso de la primera autoría. Adicionalmente, podemos mencionar que el promedio de colaboradores por artículo fue de 4.1 y que más de $50 \%$ de los investigadores que aportaron al menos un artículo a la revista cuentan con un posgrado.

Otro factor que debemos considerar es la proporción de trabajos rechazados. Para ubicar este indicador dimos seguimiento a todos los artículos recibidos durante 1998. El resultado fue que $63 \%$ de

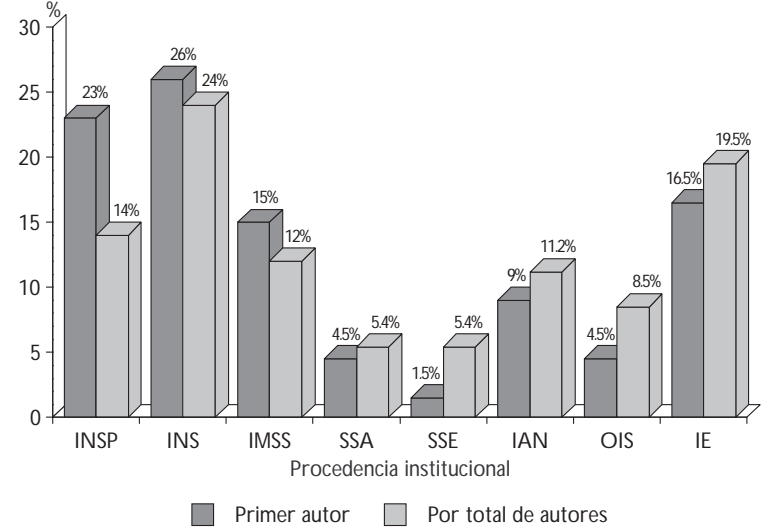

IN S: institutos nacionales de salud

SSE: servicios de salud de los estados

IAN : instituciones académicas nacionales

OIS: otras instituciones de salud

IE: instituciones extranjeras

Figura 1. Procedencia institucional de las autorías. Salud Pública de México, 1999

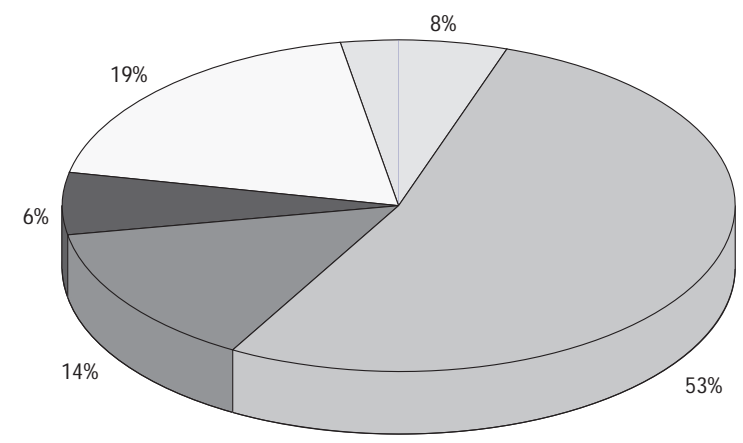

Sólo IN SP

0 tras instituciones nacionales

Instituciones extranjeras

Colaboraciones interinstitucionales sin participación de investigadores del IN SP

Colaboraciones interinstitucionales con participación de investigadores del IN SP

Figura 2. Distribución de trabajos publicados según procedencia institucional.Salud Pública de México, 1999

los trabajos recibidos en ese año fue rechazado, con lo cual alcanzamos una proporción promedio de $64 \%$ dentro del periodo 1995-1998 y de acuerdo con la evaluación que tenemos hasta ahora de los artículos so- 
metidos en 1999 esta tendencia resultará igual o mayor a $64 \%$.

Respecto a nuestra presencia internacional, cabe señalar que en los últimos años en la base de suscriptores de Salud Pública de México se han incorporado cerca de 700 bibliotecas y centros de documentación de México, América Latina, Estados Unidos de América, Canadá y Europa. Asimismo, la asistencia permanente a eventos académicos y ferias del libro durante este lapso, permitió un aumento en la demanda y un incremento en el tiraje de 2000 a 4000 ejemplares.

Para finalizar, queremos agradecer a nuestro Comité Editorial, y en especial al Consejo de Editores, el apoyo que recibimos durante este periodo para realizar satisfactoriamente nuestra labor. Este modesto reconocimiento lo hacemos extensivo, por supuesto, a autores, dictaminadores y al grupo de trabajo del área de publicaciones del INSP. 